\title{
Introduction to FUR XVII special issue
}

\author{
Ganna Pogrebna ${ }^{1,2,3}$
}

Published online: 20 April 2018

(c) Springer Science+Business Media, LLC, part of Springer Nature 2017

\section{Introduction: The FUR 2016 conference}

For over 35 years, the Foundations of Utility and Risk Conference (FUR) serves as a beacon which helps early career as well as established scientists working on research topics in the broad area of utility and risk to navigate through the complex and interdisciplinary landscape of research on individual and group behaviour. Traditionally, the FUR Conference brings together decision theorists, behavioural scientists, economists, psychologists, mathematicians, management scientists, medical and health scientists, philosophers, statisticians, and other researchers. Currently, it is one of the most prestigious conferences in decision theory and behavioural science. Over the course of the FUR history, the conference was hosted in ten countries with over 2060 papers

\footnotetext{
I am grateful to the participants, speakers, organising team, and sponsors of the 2016 Foundations of Utility and Risk Conference. I would also like to thank the international scientific committee of the FUR Conference and especially Mohammed Abdellaoui, and Peter Wakker for their support at different stages of the conference organization.
}

$凶$ Ganna Pogrebna gpogrebna@gmail.com

1 Department of Economics, Birmingham Business School, University of Birmingham, JG Smith Building, Birmingham B15 2TT, UK

2 The Alan Turing Institute, 96 Euston Road, Kings Cross, London NW1 2DB, UK

3 Warwick Manufacturing Group (WMG), Centre for Competitive Advantage in the Global Economy (CAGE), Warwick Institute for the Science of Cities (WISC), University of Warwick, Gibbet Hill Road, Coventry CV4 7AL, UK 
Fig. 1 Suggested permanent FUR logo

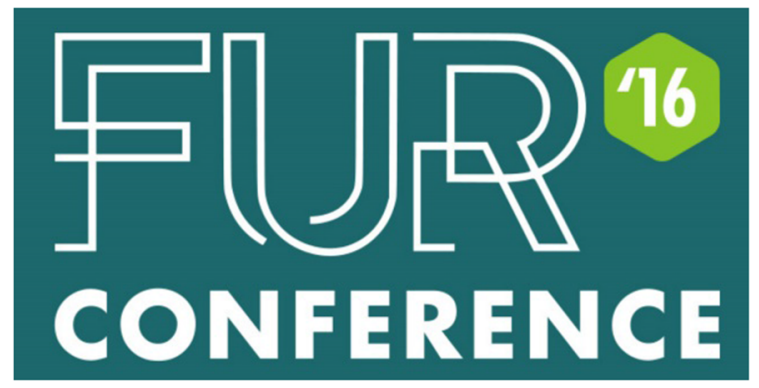

presented in total and six Nobel laureates giving talks at FUR at different points in time.

I still remember my first FUR conference which took place in Rome in 2006. That conference not only struck me by its innovative scientific programme, but also impressed me by the common spirit of scholarship among scientists who came from different disciplines and, yet, could constructively talk to one another. I have not missed a single FUR conference since. Of course, back in 2006, I could not even imagine that in 10 years' time, I would have an opportunity to organize an FUR Conference. ${ }^{1}$

I was very fortunate to be working alongside Tigran Melkonyan (Warwick Business School), Andrea Isoni (Warwick Business School), Elliot Ludvig (Department of Psychology at the University of Warwick), as well as Alexander Kharlamov (Warwick Manufacturing Group) who formed the core of the local organising team. The Conference was also supported by generous sponsorship from the Warwick Manufacturing Group, the Leverhulme Trust, Economic and Social Research Council (ESRC) Network for Integrated Behavioural Science (NIBS), Economic and Social Research Council (ESRC) Centre for Competitive Advantage in the Global Economy (CAGE) as well as Global Priorities Program in Behavioural Science at the University of Warwick. Apart from the distinguished International Conference Committee of the FUR, the local Advisory Board included such leading behavioural scientists, decision, and game theorists as Nick Chater, Graham Loomes, Zvi Zafra, Daniel Read, Peter J Hammond, and Andrew Oswald. The conference was also supported by a large technical and administrative team as well as volunteers.

Thanks to this support, the FUR 2016 conference introduced a number of important innovations such as established a permanent conference domain http://furconference. org; created a mailing list for the FUR community, suggested a permanent FUR logo (see Fig. 1), and established FUR presence on social media (Facebook and Twitter). The conference was also streamed online for audiences who wanted to connect and listen to plenary talks remotely via the Internet. ${ }^{2}$

\footnotetext{
1 The FUR 2016 Conference attracted over 400 submissions, of which, after the initial pre-screening 269 were considered for oral presentations and 54 for poster presentation. After a thorough refereeing process, 196 papers were accepted for oral and 26 for poster presentations. Overall, 267 people attended the conference including those who did not present papers.

${ }^{2}$ It is hoped that future conference organisers will change colour scheme and year of the logo keeping the FUR branding the same.
} 


\section{The FUR 2016 conference keynote speakers and round tables}

The scientific programme of the conference included talks from 3 keynote speakers (Larry Samuelson, Elke Weber, and Aldo Rustichini), 17 participants in 4 plenary roundtables, 196 presenters in parallel sessions, and 26 presenters in a poster session. Larry Samuelson (Yale University) in his keynote address "Agreeing to Disagree in Large Worlds" discussed issues at the forefront of game-theoretic research. In her talk “Preference: Primitive or Constructed Value?" Elke Weber (Princeton University) provided an overview of the main directions for decision-theoretic research in psychology. The presentation by Aldo Rustichini (University of Minnesota) "Biological Foundations of Choice Theory" offered the latest developments in establishing links between biology and neuroscience and research on risk and utility. Four roundtables covered a broad range of topics of interest to the FUR community: Ambiguity and Learning, Behavioural Game Theory, Imprecision and Noise, as well as Biological and Neuro Foundations of Utility and Risk.

The first Roundtable on Ambiguity and Learning featured interventions by Cleotilde Gonzalez (Carnegie Mellon University), Sujoy Mukerji (Queen Mary University of London), Peter Klibanoff (Northwestern University), and Aurélien Baillon (Erasmus University Rotterdam). The panel discussed recent research that crossed the borders of traditional descriptive and experiential approaches that attempted to address decision making in situations where many levels of information could be available. It also highlighted issues around the strategic use of ambiguity. Specifically, the speakers proposed to apply smooth ambiguity preferences to games of incomplete information. Roundtable panellists also talked about new methods which allowed to separate ambiguity attitudes and subjective probabilities.

The topic of the second Roundtable was Behavioural Game Theory and included talks by Robert Sugden (University of East Anglia), Rosemarie Nagel (Universitat Pomepeu Fabra), Andrew M. Colman (University of Leicester), and Nick Chater (Warwick Business School). The panel discussed recent evidence from experiments on coordination games, where explanation of observed behaviour required players to adopt multiple reasoning processes (even within the same game) and where the mode of reasoning was dependent on game parameters. The roundtable also presented new behavioural game theory models such as the virtual bargaining approach. Speakers also talked about how recently experimental economics has become a bridge for interdisciplinary collaborations across economics, biology, neuro science, psychology, and other disciplines.

John Hey (University of York), Michael H. Birnbaum (California State University, Fullerton), Michel Regenwetter (University of Illinois at Urbana-Champaign), and Graham Loomes (Warwick Business School) took part in the third Roundtable on Imprecision and Noise. This roundtable also featured a special appearance from Jerome R. Busemeyer who, for health reasons, could not attend the conference, but who has given the first-ever virtual talk at the FUR using the web-conferencing technology. Speakers at this roundtable highlighted the importance of carefully selecting the models of stochastic choice when conducting empirical analysis. They also focused on potential sources of noise and error variability in choice tasks and criteria for con- 
structing new theories of this variability. The panel also discussed new approaches to modelling noise and imprecision in individual choice.

The traditional Young Economist talk at FUR 2016 was replaced by the Young Talent Roundtable. The theme of the roundtable was Biological and Neuro Foundations of Utility and Risk. This roundtable included talks by early career researchers (all at assistant professor level) with proven track record of top-published papers: Ian Krajbich (Ohio State University), Cendri Hutcherson (University of Toronto), Joseph McGuire (Boston University), and Elliot Ludvig (University of Warwick). The panel discussed how eye-tracking technology can be used to conduct tests of reinforcement learning; provided an overview of neurally-informed computational models of choice which can account for a wide range of complex social decisions; presented behavioural evidence that decision makers re-appraise future prospects in a dynamic and contextsensitive manner and use this ongoing re-appraisal as a basis for deciding whether to sustain or curtail persistence; and showed that both people and pigeons exhibit a similar bias toward overweighting the extreme outcomes (biggest win and biggest loss) in experience-based risky choice tasks.

\section{Conclusion: current trends and new frontiers in research on utility and risk}

The FUR conference has traditionally covered a wide range of areas including decision theory, behavioural game theory, models of stochastic choice, behavioural/experimental economics and psychology, judgement and decision making, social psychology, neuroscience, to name a few. The FUR 2016 conference was not an exception: it featured a wide range of (cross-disciplinary) topics. From the conference programme, it emerged that ambiguity remained one of the main research areas of interest to the FUR community. Of 49 parallel sessions, 9 featured theoretical, and empirical work on ambiguity. Specifically, there were four sessions on Experiments and Ambiguity, two on Updating and Ambiguity, two on Theory and Applications of Ambiguity, and one on Ambiguity in Games. Other popular themes of the conference were Risk Attitudes (3 of 49 sessions), Intertemporal Choice (3 of 49 sessions), Intelligence and Strategic Behaviour (2 of 49 sessions), Games and Strategic Behaviour (2 of 49 sessions), and Field Experiments ( 2 of 49 sessions). Research on these topics has long history at the FUR and it seems that it becomes more and more prevalent as well as developed.

At the same time, apart from traditional themes, the FUR 2016 conference featured several topics which were not presented at the FUR before. Particularly, the FUR 2016 highlighted research on decision theory and Big Data as a major new frontier for the future. In a parallel session on Big Data, researchers discussed how privacy of personal data in the new digital economy should be modelled and analysed using decisiontheoretic and game-theoretic methodology; how existing models of individual decision making could be tested using large data sets; and how models of imprecision and noise may potentially enrich machine learning algorithms.

This Special Issue captures the main trends in utility and risk research which emerged at the FUR 2016 conference. Two papers enhance our understanding of ambiguity investigating ambiguity aversion under maximum-likelihood updating and 
strategic ambiguity in games. Emotions and their meaning in different decision-making situations are explored in two papers on curse of hope and health-belief formation. Two papers provide further insights into measurement and analysis of risk attitudes by addressing the issues in search problems and applying multi-choice elicitation techniques. The Issue also covers such themes as confidence and learning using Bayesian decision mechanisms and belief formation in signalling games.

The remainder of this Special Issue is organised as follows. The Special Issue opens with a contribution by Orestis Kopsacheilis titled "The Role of Information Search and Its Influence on Risk Preferences" discussing risk attitudes and information search in decision by experience. It is followed by the paper by Giuseppe Attanasi, Nikolaos Georgantzís, Valentina Rotondi, and Daria Vigani titled "Lotteryand Survey-based Risk Attitudes Linked through a Multi-choice Elicitation Task" looking at risk attitudes measures across lottery-based and survey-based individual choices. The third paper in this Special Issue is an article by Daniel Heyen titled "Ambiguity Aversion under Maximum Likelihood Updating" providing an example in which maximum-likelihood updating induces an ambiguity-averse max-min expected utility. It is followed by "Strategic Ambiguity and Decision-making: An Experimental Study", a contribution by Sara le Roux and David Kelsey, exploring the differences between the effect of ambiguity in individual decision-making problems and in games. The Special Issue then continues with an article "The Impact of Health Related Emotions on Beliefs Formation and Behavior" by Elyès Jouini and Clotilde Napp where emotions are introduced into modelling health-related decision making. The 6th paper in the Special Issue is "The Curse of Hope" by Serge Macé and Fabrice Le Lec. It extends reference-dependent model of preferences by adding the individual's current reference point (status quo) to the stochastic reference point. The penultimate contribution of the Special Issue is titled "Confidence Biases and Learning among Intuitive Bayesians". Authors Louis Levy-Garboua, Muniza Askari, and Marco Gazel explore why intuitive Bayesians make systematically wrong predictions of their own performance. The Special Issue concludes with a paper by Alex Possajennikov titled "Belief Formation in a Signaling Game without Common Prior: An Experiment" where the author shows that elicitation of beliefs does not considerably change the pattern of play in the game. 